\title{
Ground simulation research on the super large moment of inertia of the space transfer manipulator
}

\author{
Wei Zhang ${ }^{1, *}$, Hongxin Wang ${ }^{1}$, Weijian Jia ${ }^{1}$, Lin Ding ${ }^{1}$, and Ying Cheng ${ }^{1}$ \\ ${ }^{1}$ Locomotive College of West Anhui University, Lu'an City, Anhui Province, China
}

Keywords: Space Manipulator, Large moment of inertia, Ground simulation, Dynamic model, ADAMS.

\begin{abstract}
During the construction of the space station, the cabin is driven by the transposition manipulator in space. Because of the large mass and volume of the cabin, which is not less than 22 tons, there will be emerge a large moment of inertia in the transposition process, which is not less than . Therefore, a full physical experimental method is designed to simulate the moment of inertia of the transposition manipulator in space on the ground. In this paper, the three-dimensional model of the whole physical loading method is built, and the dynamic model is built by using the Lagrange equilibrium method. The minimum values of the moment of inertia applied by this method to the shoulder joint and the wrist joint of the space manipulator are calculated as and respectively. The simulation through Adams show that the error of the method to the shoulder joint and the wrist joint of the manipulator is increased by $4.4 \%$ and $5.59 \%$ respectively compared with that of the dynamics, that is to say no matter in numerical value or error, all physical experiment methods meet the requirements of ground test.
\end{abstract}

\section{Introduction}

China plans to build and operate the near earth space station in 2022 [1]. The space station is a "building block" assembly structure, which is composed of core module, experimental module I, experimental module II, cargo spacecraft and manned spacecraft [2](see Fig. 1-1 ). The space manipulator is a two joint three-stage structure. The two joints are respectively equipped with drive motors to control the movement of the manipulator. The two manipulators are respectively referred to as shoulder joint and wrist joint, and their models are shown in Figure 1-2.

\footnotetext{
*Corresponding author: zhangwei1055@126.com
} 


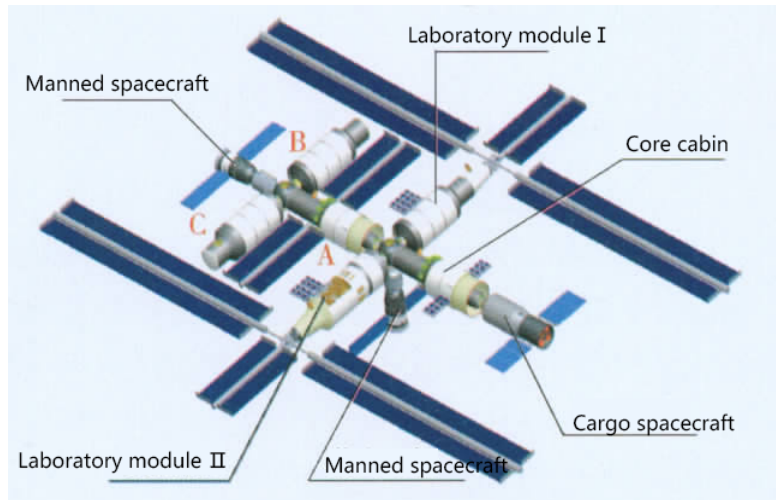

Fig. 1-1. Space station.

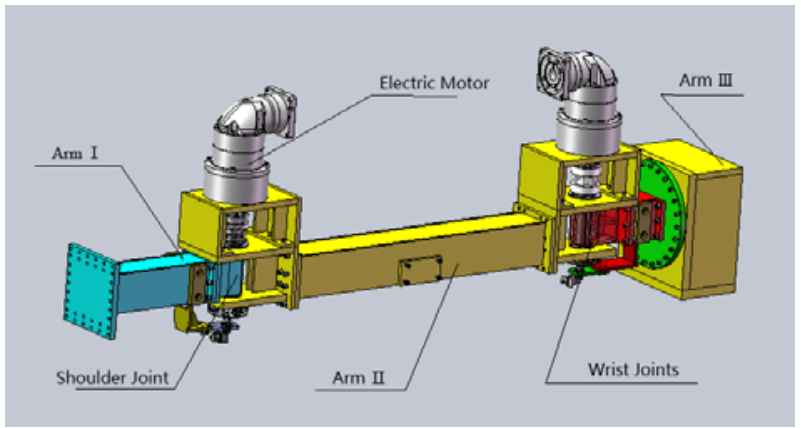

Fig. 1-2. Space manipulator model.

The working form of the space manipulator is as follows: during the construction of the space station, the core cabin is launched first, and then the experimental cabin. Due to some complex reasons, the docking between the core cabin and the experimental cabin can only be carried out in the axial direction. After the automatic docking between the experimental cabin and the core cabin is completed in the axial direction of the core cabin, the space manipulator installed on the core cabin receives the motion command. The motion refers the command will drive the motor movement installed at the shoulder joint and wrist joint of the space mechanical arm, and the space rotation mechanical arm installed on the core cabin will complete the capture of the experimental cabin under the corresponding command. According to the command, the experimental cabin connected to the axial position of the core cabin will be rotated to the radial position of the core cabin, so as to reciprocate and finally complete the construction of the space station [3,4,5], as shown in Figure 1-3

In the process of rotation, although the experimental cabin is only subjected to small gravity in space, its mass and volume are huge, and its mass is about $22000 \mathrm{~kg}$ [7], as shown in Figure 1-4, which leads to the moment of inertia of the space manipulator when it is driven to rotate. Due to the particularity of space products, it is necessary to conduct sufficient experiments on the ground for the space manipulator,In particular, the bearing capacity of the moment of inertia of the driving motor at the shoulder joint and wrist joint ensures that it is competent for the transposition task in space, and finally completes the construction of the space station. 


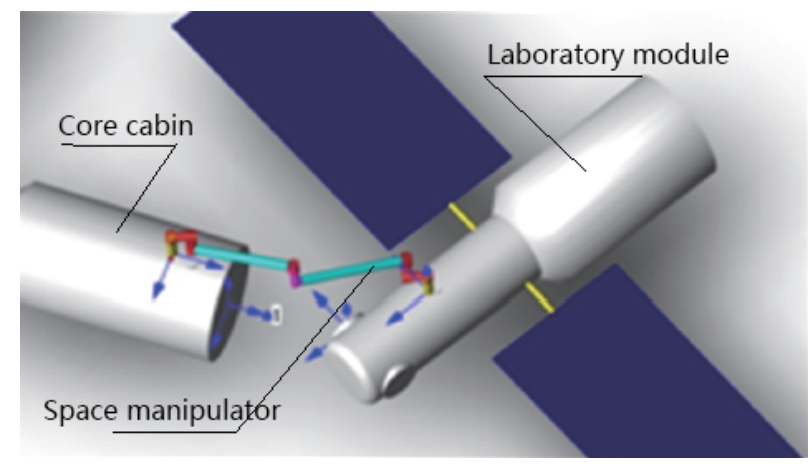

Fig. 1-3. Motion of space manipulator [6].

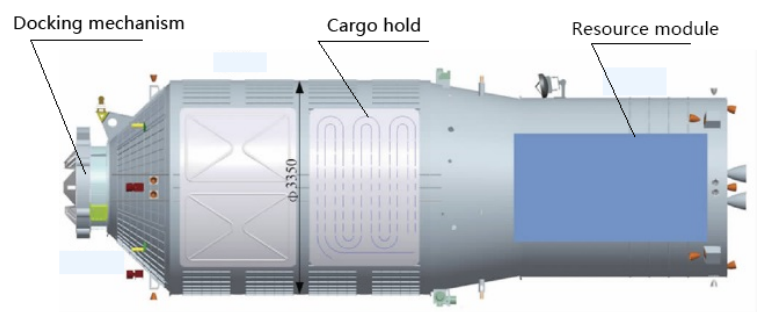

Fig. 1-4. Cargo ship.

Because of the large volume and mass of the cabin, it is difficult to simulate such a large moment of inertia on the ground.For this reason, Xu Huamin of Tsinghua University used the method of weight guide to solve the problem of moment of inertia under the condition of inertia[8]. This method proposed a new method for solving the moment of inertia, but did not design the moment of inertia model.Shen Wenbo of AVIC uses an aerocentrifuge to realize large inertia loading[9]. This method is suitable for smaller spacecraft, not for larger spacecraft such as space station.Halle. Designed a two axis inertia simulation device based on the gear train[10]. The response speed of the gear train is fast, but the generated force and torque can not be completely applied on the space rotation manipulator.Wenhao. Designed a kind of moment of inertia loading device based on flywheel[11]. Because of the inertia of flywheel, the accuracy of moment of inertia in simulation value and time is poor.Wang Wanjun . Used electric inertia to simulate the load moment of inertia[12], the torque produced by the motor has hysteresis, and the simulated moment of inertia will have some differences in time accuracy. Based on the comparison and analysis of the advantages and disadvantages of the existing large moment of inertia loading methods, a full physical experimental method is proposed for the ground experiment of the space rotation manipulator.

\section{Principle of total physical moment of inertia loading method}

In the whole physical experiment method, the inertia simulation mechanism is designed to simulate the moment of inertia of the space manipulator when it is rotated. The inertia simulation mechanism of the experimental cabin is composed of mass blocks which are connected by bolts and installed on the mounting plate, and finally constitute a $7000 \mathrm{~kg}$ inertia simulation mechanism. In this way, the inertia simulation mechanism follows the rotation process of the manipulatorThe corresponding moment of inertia is applied to the mechanical arm. 
For the microgravity environment of the manipulator in space, the air floatation method is used to provide microgravity environment for the whole physical experimental device. Different air floatation mechanisms are designed for the moment of inertia simulation mechanism, the space rotation manipulator and the intermediate connecting beam. For the inertia simulation mechanism with a weight of $7000 \mathrm{~kg}$, the air floatation plate with the overall dimension is designed to provide microgravity for it. For the space rotation machineryAn anti gravity mechanism based on air feet is designed to provide microgravity environment for the arm and the middle connecting beam.

The full physical experimental device works in the ground microgravity environment where the air flotation platform provides the whole experimental device with simulated space gravity. The space manipulator rotates according to the orbit of rotation in space, and the inertia simulation mechanism is at the end of the experimental device. It is connected with the manipulator through the middle beam, and the space manipulator rotates according to the predetermined program in space,Because the relative gravity and friction force in microgravity environment can be ignored, the inertia simulation mechanism will follow the manipulator to rotate. In this process, the moment of inertia of the rotating manipulator in space is generated by simulation. In the whole experimental process, the power source of the whole physical method is from the driving mechanism at the shoulder joint and wrist joint of the space rotating manipulatorThere is no other power source introduced, so the data simulated by the full physical method is full true simulation in terms of accuracy and reliability. The model of the whole device is shown in Figure 2-1.

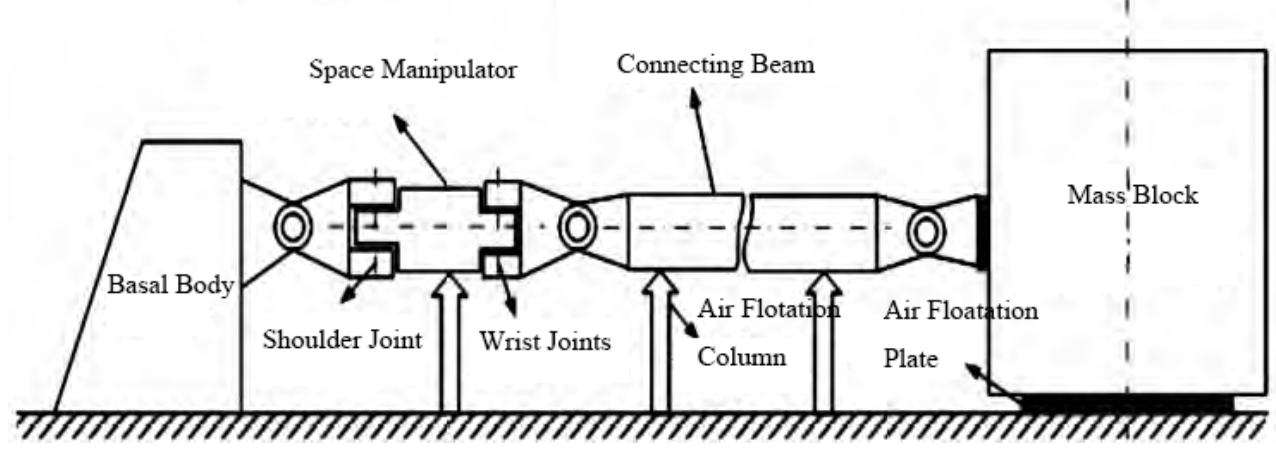

Fig. 2-1. Principle of full physical experiment[13].

\section{Dynamic modeling of total physical moment of inertia loading method}

In the whole physical experiment device, the moment of inertia of the space rotation manipulator mainly comes from the moment of inertia generated when the space rotation manipulator rotates itself and the moment of inertia applied by the inertia simulation mechanism to the manipulator when it follows the rotation

(1) the manipulator itself, because of its mass, will produce the corresponding moment of inertia to the shoulder joint and wrist joint as long as the manipulator is rotating, whether in outer space or in the whole physical test.

(2) inertia simulation device, this part is added artificially in this test, in order to simulate the moment of inertia produced by the outer space experimental cabin when the mechanical arm is rotating with it.In outer space, it is the moment of inertia of the experimental cabin, 
while in this whole physical experiment, it is the moment of inertia generated by the human external mass in the process of rotation.

\subsection{Dynamic equation of manipulator}

Lagrangian equilibrium method is used to model the dynamics of the space transfer manipulator. Lagrangian function $\mathrm{L}$ is defined as the difference between kinetic energy $\mathrm{k}$ and potential energy $\mathrm{P}$ of the system[14,15], namely:

$$
L=K-P
$$

$\mathrm{K}$ and $\mathrm{P}$ in the formula can be expressed in any convenient coordinate system. The Lagrange equation, that is, the dynamic equation of the system, is expressed as follows:

$$
\mathbf{F}_{i}=\frac{d}{d t} \frac{\partial L}{\partial \dot{q}_{i}}-\frac{\partial L}{\partial q_{i}}, i=1,2, \cdots, n
$$

In the formula, the force or moment is determined by the linear coordinate or the angle coordinate, obviously the speed. Although the mechanical arm studied in this paper is a three link mechanical arm, it can be simplified as a two link mechanical arm because the shoulder joint is fixed on the core cabin[16], which can be simplified as shown in Figure 3-1. $\mathbf{F}_{i}$

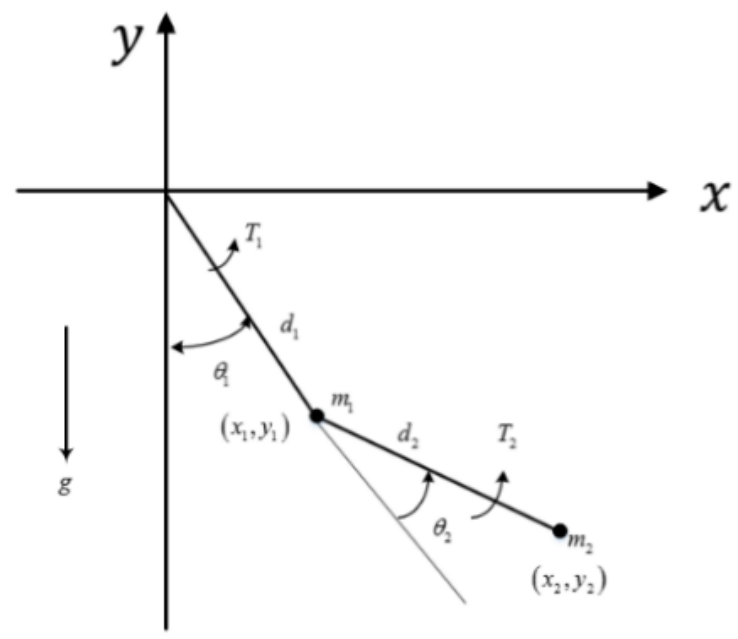

Fig. 3-1. Dynamics simplification of manipulator.

where and are the masses of connecting rod 1 and 2, and are the lengths of connecting rod 1 and 2 respectively, and are generalized coordinates, and $\mathrm{G}$ is the acceleration of gravity.

(1) calculate the kinetic energy and potential energy of connecting rod 1.Because:

$$
\begin{gathered}
K_{1}=\frac{1}{2} m_{1} v_{1}^{2} \\
P_{1}=m_{1} g h_{1}
\end{gathered}
$$

From Figure 3-1, we can get: 


$$
\begin{aligned}
& K_{1}=\frac{1}{2} m_{1} d_{1}^{2} \dot{\theta}_{1}^{2} \\
& P_{1}=-m_{1} g d_{1} \cos \theta_{1}
\end{aligned}
$$

(2) calculate the kinetic energy sum of connecting rod 2

$$
\begin{gathered}
K_{2}=\frac{1}{2} m_{2} v_{2}^{2} \\
P_{2}=m g y_{2}
\end{gathered}
$$

According to Figure (3-1):

$$
\begin{aligned}
& x_{2}=d_{1} \sin \theta_{1}+d_{2} \sin \left(\theta_{1}+\theta_{2}\right) \\
& \mathrm{y}_{2}=-d_{1} \cos \theta_{1}-d_{2} \cos \left(\theta_{1}+\theta_{2}\right)
\end{aligned}
$$

There are:

$$
\begin{aligned}
& \dot{x}_{2}=d_{1} \dot{\theta}_{1} \cos \theta_{1}+d_{2}\left(\dot{\theta}_{1}+\dot{\theta}_{2}\right) \cos \left(\theta_{1}+\theta_{2}\right) \\
& \dot{\mathrm{y}}_{2}=d_{1} \dot{\theta}_{1} \sin \theta_{1}+d_{2}\left(\dot{\theta}_{1}+\dot{\theta}_{2}\right) \sin \left(\theta_{1}+\theta_{2}\right)
\end{aligned}
$$

As shown in Figure 3-1:

$$
v_{2}^{2}=\dot{x}_{2}^{2}+\dot{y}_{2}^{2}
$$

Take formula (11) (12)into formula (13) to get:

$$
v_{2}^{2}=d_{1}^{2} \dot{\theta}_{1}^{2}+2 d_{1} d_{2} \dot{\theta}_{1}\left(\dot{\theta}_{1}+\dot{\theta}_{2}\right) \cos \theta_{2}+d_{2}^{2}\left(\dot{\theta}_{1}+\dot{\theta}_{2}\right)^{2}
$$

Take formula (11), formula (12) and formula (14) into formula (7) and formula (8) respectively to get:

$$
\begin{aligned}
& K_{2}=\frac{1}{2} m_{2}\left(d_{1}^{2} \dot{\theta}_{1}^{2}+2 d_{1} d_{2} \dot{\theta}_{1}\left(\dot{\theta}_{1}+\dot{\theta}_{2}\right) \cos \theta_{2}+d_{2}^{2}\left(\dot{\theta}_{1}+\dot{\theta}_{2}\right)^{2}\right) \\
& P_{2}=-m g\left(d_{1} \cos \theta_{1}+d_{2} \cos \left(\theta_{1}+\theta_{2}\right)\right)
\end{aligned}
$$

The total kinetic energy and potential energy of the two link manipulators can be obtained as follows:

$$
\begin{array}{r}
K=K_{1}+K_{2}=\frac{1}{2} m_{1} d_{1}^{2} \dot{\theta}_{1}^{2}+\frac{1}{2} m_{2}\left(d_{1}^{2} \dot{\theta}_{1}^{2}+d_{2}^{2}\left(\dot{\theta}_{1}+\dot{\theta}_{2}\right)^{2}\right)+m_{2} d_{1} d_{2} \dot{\theta}_{1}\left(\dot{\theta}_{1}+\dot{\theta}_{2}\right) \cos \theta_{2} \\
P=p_{1}+P_{2}=-\left(m_{1}+m_{2}\right) g d_{1} \cos \theta_{1}-m_{2} g d_{2} \cos \left(\theta_{1}+\theta_{2}\right)
\end{array}
$$

Here, take formula (17) and formula (18) into formula (1) to get: 
$L=K-P=\frac{1}{2} m_{1} d_{1}^{2} \dot{\theta}_{1}^{2}+\frac{1}{2} m_{2} d_{1}^{2} \dot{\theta}_{1}^{2}+\frac{1}{2} m_{2} d_{2}^{2}\left(\dot{\theta}_{1}+\dot{\theta}_{2}\right)^{2}+\left(m_{1}+m_{2}\right) g d_{1} \cos \theta_{1}+m_{2} g d_{2} \cos \left(\theta_{1}+\theta_{2}\right)+m_{2} d_{1} d_{2} \dot{\theta}_{1}\left(\dot{\theta}_{1}+\dot{\theta}_{2}\right) \cos \theta_{2}$

The derivative and partial derivative of formula (19) are as follows:

$$
\begin{aligned}
& \frac{\partial L}{\partial \theta_{1}}=-\left(m_{1}+m_{2}\right) g d_{1} \sin \theta_{1}-m_{2} g d_{2} \sin \left(\theta_{1}+\theta_{2}\right) \\
& \frac{\partial L}{\partial \theta_{2}}=-m_{2} d_{1} d_{2} \dot{\theta}_{1}\left(\dot{\theta}_{1}+\dot{\theta}_{2}\right) \sin \theta_{2}-m_{2} g d_{2} \sin \left(\theta_{1}+\theta_{2}\right) \\
& \frac{\partial L}{\partial \dot{\theta}_{1}}=\left(m_{1}+m_{2}\right) d_{1}^{2} \dot{\theta}_{1}+m_{2} d_{2}^{2}\left(\dot{\theta}_{1}+\dot{\theta}_{2}\right)+m_{2} d_{1} d_{2}\left(2 \dot{\theta}_{1}+\dot{\theta}_{2}\right) \cos \theta_{2} \\
& \frac{\partial L}{\partial \dot{\theta}_{2}}=m_{2} d_{1} d_{2} \dot{\theta}_{1} \cos \theta_{2}+m_{2} d_{2}^{2}\left(\dot{\theta}_{1}+\dot{\theta}_{2}\right)
\end{aligned}
$$

as well as

$$
\begin{gathered}
\frac{d}{d t} \frac{\partial L}{\partial \dot{\theta}_{1}}=\left[\left(m_{1}+m_{2}\right) d_{1}^{2}+m_{2} d_{2}^{2}+2 m_{2} d_{1} d_{2} \cos \theta_{2}\right] \ddot{\theta}_{1}+\left(m_{2} d_{2}^{2}+m_{2} d_{1} d_{2} \cos \theta_{2}\right) \ddot{\theta}_{2}-2 m_{2} d_{1} d_{2} \dot{\theta}_{1} \dot{\theta}_{2} \sin \theta_{2}-m_{2} d_{1} d_{2} \dot{\theta}_{2}^{2} \sin \theta_{2} \\
\frac{d}{d t} \frac{\partial L}{\partial \dot{\theta}_{2}}=m_{2} d_{1} d_{2}\left(\ddot{\theta}_{1} \cos \theta_{2}-\dot{\theta}_{1} \dot{\theta}_{2} \sin \theta_{2}\right)+m_{2} d_{2}^{2}\left(\ddot{\theta}_{1}+\ddot{\theta}_{2}\right)
\end{gathered}
$$

Take the corresponding derivative into equation (2) to get:

$$
\begin{aligned}
T_{1} & =\frac{d}{d t} \frac{\partial L}{\partial \dot{\theta}_{1}}-\frac{\partial L}{\partial \theta_{1}} \\
& =\left[\left(m_{1}+m_{2}\right) d_{1}^{2}+m_{2} d_{2}^{2}+2 m_{2} d_{1} d_{2} \cos \theta_{2}\right] \ddot{\theta}_{1}+\left(m_{2} d_{2}^{2}+m_{2} d_{1} d_{2} \cos \theta_{2}\right) \ddot{\theta}_{2}-2 m_{2} d_{1} d_{2} \dot{\theta}_{1} \dot{\theta}_{2} \sin \theta_{2}+m_{2} g d_{2} \sin \left(\theta_{1}+\theta_{2}\right)-m_{2} d_{1} d_{2} \dot{\theta}_{2}^{2} \sin \theta_{2}+\left(m_{1}+m_{2}\right) g d_{1} \sin \theta_{1}
\end{aligned}
$$

$$
T_{2}=\frac{d}{d t} \frac{\partial L}{\partial \dot{\theta}_{2}}-\frac{\partial L}{\partial \theta_{2}}=m_{2} d_{2}^{2}\left(\ddot{\theta}_{1}+\ddot{\theta}_{2}\right)+m_{2} d_{1} d_{2}\left(\ddot{\theta}_{1} \cos \theta_{2}-\dot{\theta}_{1} \dot{\theta}_{2} \sin \theta_{2}\right)+m_{2} d_{1} d_{2} \dot{\theta}_{1}\left(\dot{\theta}_{1}+\dot{\theta}_{2}\right) \sin \theta_{2}+m_{2} g d_{2} \sin \left(\theta_{1}+\theta_{2}\right)
$$

Here, formula (26) and formula (27) are written in the form of equation as follows:

$$
\begin{aligned}
& T_{1}=D_{11} \ddot{\theta}_{1}+D_{12} \ddot{\theta}_{2}+D_{111} \dot{\theta}_{1}^{2}+D_{122} \dot{\theta}_{2}^{2}+D_{112} \dot{\theta}_{1} \dot{\theta}_{2}+D_{121} \dot{\theta}_{2} \dot{\theta}_{1}+D_{1} \\
& T_{2}=D_{21} \ddot{\theta}_{1}+D_{22} \ddot{\theta}_{2}+D_{211} \dot{\theta}_{1}^{2}+D_{222} \dot{\theta}_{2}^{2}+D_{212} \dot{\theta}_{1} \dot{\theta}_{2}+D_{221} \dot{\theta}_{2} \dot{\theta}_{1}+D_{2}
\end{aligned}
$$

It can be further written in the form of matrix:

$$
\left[\begin{array}{l}
T_{1} \\
T_{2}
\end{array}\right]=\left[\begin{array}{ll}
D_{11} & D_{12} \\
D_{21} & D_{22}
\end{array}\right]\left[\begin{array}{l}
\ddot{\theta}_{1} \\
\ddot{\theta}_{2}
\end{array}\right]+\left[\begin{array}{cc}
D_{111} & D_{122} \\
D_{211} & D_{222}
\end{array}\right]\left[\begin{array}{c}
\dot{\theta}_{1}^{2} \\
\dot{\theta}_{2}^{2}
\end{array}\right]+\left[\begin{array}{cc}
D_{112} & D_{121} \\
D_{212} & D_{221}
\end{array}\right]\left[\begin{array}{c}
\dot{\theta}_{1} \dot{\theta}_{2} \\
\dot{\theta}_{2} \dot{\theta}^{\prime}
\end{array}\right]+\left[\begin{array}{c}
D_{1} \\
D_{2}
\end{array}\right]
$$

In the formula, it is called the effective inertia of joint I, because the acceleration of joint $\mathrm{J}$ will produce an inertia force equal to on joint I; it is called the coupling inertia between joint 
I and $\mathrm{j}$, because the acceleration of joint $\mathrm{I}$ and $\mathrm{j}$ and will produce an inertia force equal to or on joint I or joint $\mathrm{J}$; the term is the centripetal force generated on joint I by the velocity of joint $\mathrm{J}$; it is the effect caused by the velocity of joint $\mathrm{J}$ and joint KCoriolis force on the joint; represents the gravity at joint $\mathrm{I}$.

The comparison formula (26) (27) (28) and (29) show that:

$$
\begin{aligned}
& D_{11}=\left(m_{1}+m_{2}\right) d_{1}^{2}+m_{2} d_{2}^{2}+2 m_{2} d_{1} d_{2} \cos \theta_{2} \\
& D_{22}=m_{2} d_{2}^{2}
\end{aligned}
$$

The coupling between them

$$
D_{12}=m_{2} d_{2}^{2}+m_{2} d_{1} d_{2} \cos \theta_{2}
$$

Centripetal acceleration coefficient:

$$
\begin{aligned}
& D_{111}=0 \\
& D_{122}=-m_{2} d_{1} d_{2} \sin \theta_{2} \\
& D_{122}=-m_{2} d_{1} d_{2} \sin \theta_{2} \\
& D_{122}=-m_{2} d_{1} d_{2} \sin \theta_{2} \\
& D_{222}=0
\end{aligned}
$$

Coriolis acceleration coefficient:

$$
\begin{aligned}
& D_{112}=-m_{2} d_{1} d_{2} \sin \theta_{2} \\
& D_{121}=-m_{2} d_{1} d_{2} \sin \theta_{2} \\
& D_{212}=0 \\
& D_{221}=0
\end{aligned}
$$

Gravity term:

$$
\begin{aligned}
& D_{1}=\left(m_{1}+m_{2}\right) g d_{1} \sin \theta_{1}+m_{2} g d_{2} \sin \left(\theta_{1}+\theta_{2}\right) \\
& D_{2}=m_{2} g d_{2} \sin \left(\theta_{1}+\theta_{2}\right)
\end{aligned}
$$


The moment of inertia produced by the mass block in the following process is the most important part of the moment of inertia in this test. As shown in Figure 2-4 in Chapter 2, the mass block of the inertia simulation mechanism consists of 12 weight blocks with a total weight of 7 tons. The length $\times$ width $\times$ height of a single weight block $=1200 \mathrm{~mm} \times 570 \mathrm{~mm}$ $\times 100 \mathrm{~mm}$. The moment of inertia at any point is as follows according to the formula:

$$
\begin{aligned}
J_{e} & =\sum m_{i} d_{i}^{2} \\
& =\sum m_{i}\left[r_{i}^{2}-\left(\overrightarrow{\mathrm{e}} \cdot \vec{r}_{i}\right)\left(\vec{r}_{i} \cdot \vec{e}\right)\right] \\
& =\sum m_{i}\left[e^{T} r_{i}^{2} E e-e^{T} r_{i} r_{i}^{T} e\right] \\
& =e^{T} \sum m_{i}\left[r_{i}^{2} E-r_{i} r_{i}^{T}\right] e
\end{aligned}
$$

According to the composition of the full physical test in Figure 2-1, we can see the position of the shoulder joint and wrist joint of the tested mechanical arm. According to the principle of the test, we can know that the distance between the shoulder joint and the mass block of the tested mechanical arm is always the same as the length from the middle connecting mechanism to the end of the shoulder joint, no matter how the mechanical arm rotates, the distance does not change, soThe moment of inertia of the shoulder joint caused by the mass block is a constant fixed value.Compared with the fixed distance between the shoulder joint and the mass, the distance between the mass and the wrist joint changes with the rotation of the manipulator.Through the software, it can be analyzed that in the process of following the rotation of the mass block, the minimum value of the rotation radius is $9800.8 \mathrm{~mm}$, and the maximum radius is $11451 \mathrm{~mm}$, so that the minimum value of the moment of inertia at the shoulder joint of the space rotation manipulator is, and the minimum value of the moment of inertia at the wrist joint is.

\section{Simulation of total physical moment of inertia loading method}

In order to further verify the feasibility of the whole physical experiment, the 3D model established by solid works is imported into Adams to obtain the moment of inertia of the space mechanical arm shoulder joint and wrist joint which can be loaded by the whole physical experiment method.The distance between the following mass and the wrist joint is a variable value for the rotation of the manipulator.Through the Adams[17] model analysis of the whole physical experiment, the motion track of the whole following mass block is obtained, as shown in Figure 4-1.Then import these data into Matlab[18] to analyze the accurate values, and the results are shown in Figure 4-2.

\subsection{Moment of inertia of the wrist joint of the spatial transposition mechanism}

By analyzing the ADAMS simulation results, the minimum moment of inertia of the wrist joint can be obtained, as shown in Figure 4-3.

The result of ADAMS simulation is $4.4 \%$ higher than that of dynamic modeling. 


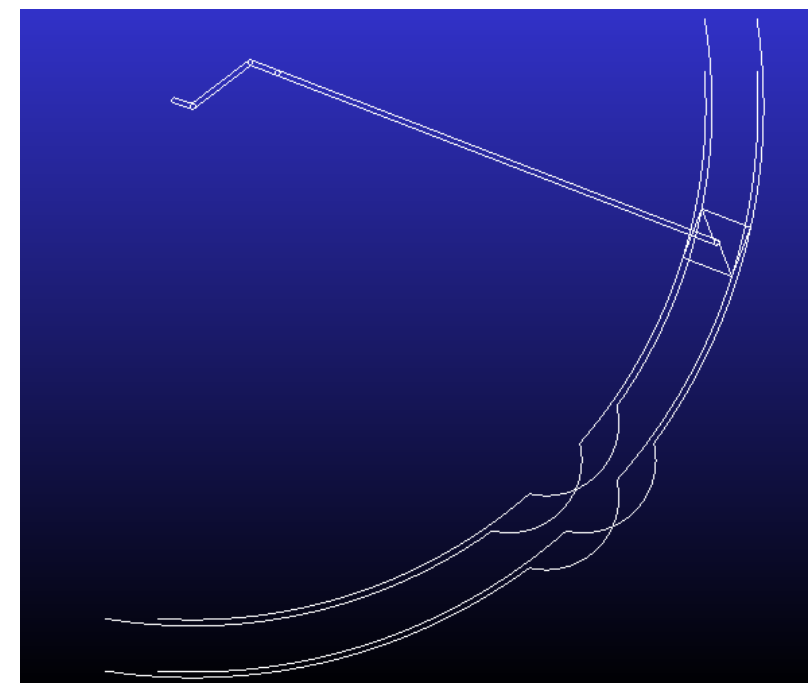

Fig. 4-1. Adam model.

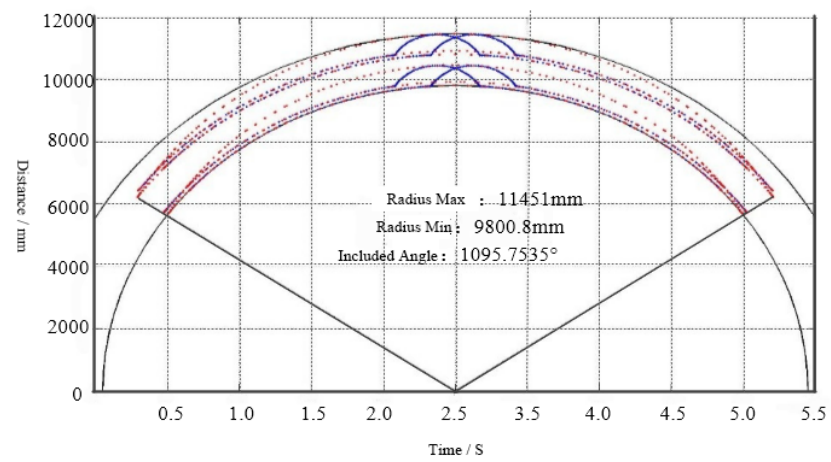

Fig. 4-2. Trajectory of manipulator.

\subsection{The moment of inertia of the shoulder joint of the space transfer manipulator}

Similarly, by analyzing the ADAMS simulation results, we can get the moment of inertia exerted by the fast mass on the shoulder joint. The minimum value is as shown in Figure 4-4.

The main reason is that the distance between the mass block and the shoulder joint changes with the rotation of the spatial rotation manipulator. Compared with the minimum value of the dynamic modeling of the shoulder joint, the ADAMS simulation increases the minimum value by $5.59 \%$. 


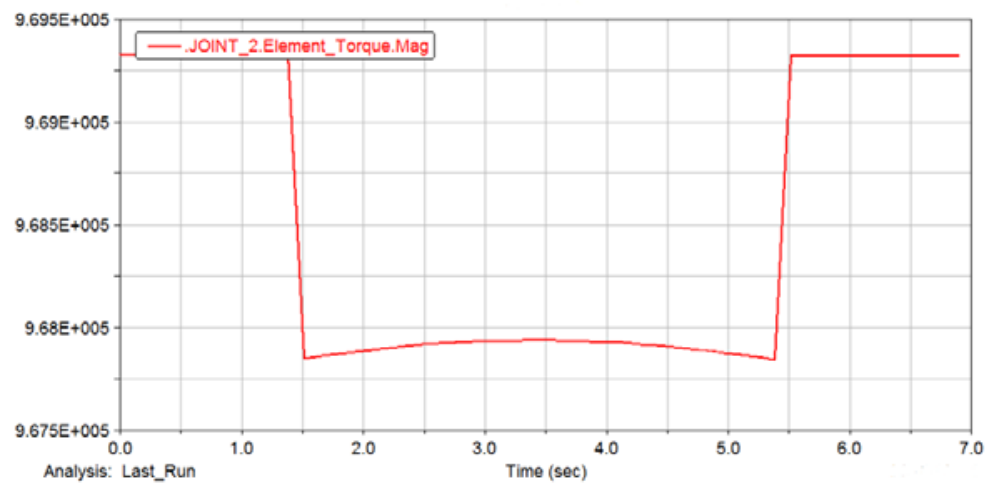

Fig. 4-3. Moment of inertia of wrist joint.

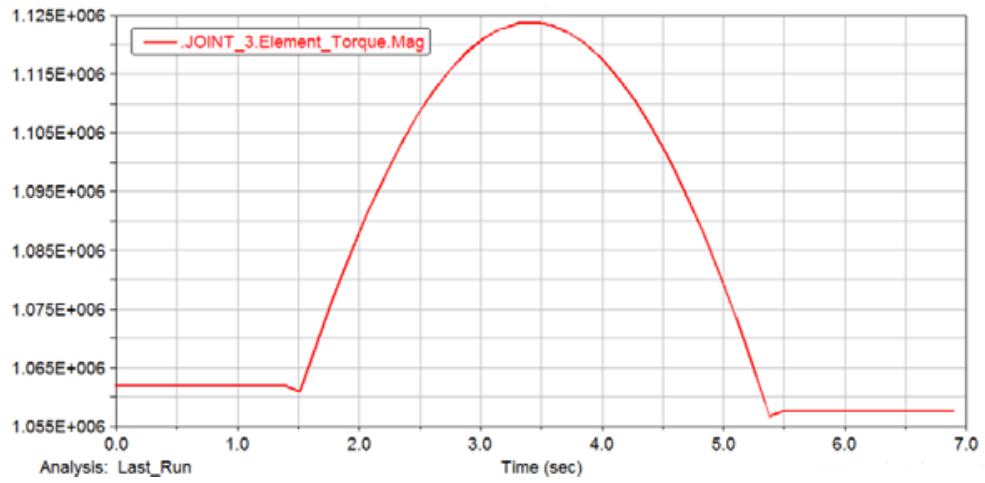

Fig. 4-4/ Moment of inertia caused by mass to wrist joint.

\section{Summary}

The super large moment of inertia of the space rotation manipulator is not less than, and it is tested on the ground. By comparing the electric inertia loading method based on the motor, the semi physical moment of inertia loading method based on the gear and the semi physical moment of inertia loading method based on the flywheel, a kind of moment of inertia loading method based on the inertia simulation mechanism is proposedIt is an all physics experiment method.

In this paper, the three-dimensional model of the whole physical loading method is built, and the dynamic model of the whole physical experimental device is established by using the Lagrange method, and the theoretical moment of inertia is calculated. The minimum moment of inertia applied to the wrist joint and the minimum moment of inertia applied to the shoulder joint by this method are calculated. Finally, the minimum moment of inertia applied to the wrist joint by this method is simulated by ADAMS softwareThe minimum moment of inertia applied at.Both of them are larger than the experimental requirements in numerical value, and the errors are $4.4 \%$ and $5.59 \%$ respectively, which can also meet the experimental requirements, that is, the full physical experimental method is effective.

Many people have offered me valuable help in my thesis writing, including my President, Director and Colleagues.

First of all, I would like to thank President Hongxin WANG for her guidance in the writing process. I can say that without the urging of President Wang, there would be no publication of this paper. 
Secondly, I would like to thank Dr. Lin DING for his great help in writing and typesetting of English papers. At the same time, I would also like to thank director Weijian JIA and Ms. Ying CHENG for their help in life and work durning writing, Thanks again for their help!

Fund projects:Application for school level natural science research project of West Anhui University

\section{Reference}

1. Xiao Jianjun, Qi Xiaojun. China\&apos;s manned space engineering enters the era of space station [J]. International space, 2018, 478 (10): 35-39

2. Zhou Jianping. General concept of China\&apos;s space station engineering [J]. Manned space flight, 2013, 19 (2): 1-10

3. Chen Shanguang, Chen Jindun, Jiang Guohua. Achievements in manned space flight and construction of space station in China $[\mathrm{J}]$. Aerospace medicine and medical engineering, 2012 (06): 5-10

4. Li Guangxing, Xiao Yuzhi, bu Shaohua, et al. Study on attitude control scheme of space station assembly process [J]. Human spaceflight, 2012, 18 (1): 22-29

5. Hu Xueping, Shi Junwei, Shen Xiaopeng. Dynamic matching design and Simulation of space station cabin transfer test system [J]. Manned space flight, 2018

6. Zhang Dawei, Liang Changchun, Wei Qingqing, et al. Trajectory tracking control and accuracy analysis of manipulator auxiliary cabin [J]. Manned space flight, 2014 (2): 104-109

7. Xu Jing. The opening of the space station\&apos;s core cockpit head shows the strength of our country [J]. China aerospace, 2018, 487 (11): 23-25

8. Xu Huamin, Guan Liwen, Wang Liping. Topological optimization of boom structure of flight simulator under inertial load [J]. Journal of mechanical engineering, 2014, 50 (9): 14-23

9. Shen Wenbo, Wang Xinlun. Design and implementation of control system for high inertia and high dynamic air centrifuge [J]. Measurement and control technology, 2015, $34(5)$

10. Halle, Fang Lijin, Xu Zhigang. Application Research of super large inertia simulation method in space transposition device [J]. Mechanical design, 2014, 31 (12): 21-25

11. Wen Hao, Jin Dongping, Chen ti. Acceleration flywheel inertia simulator and the method of using acceleration flywheel inertia simulator to simulate the rotational inertia and translational inertia of spacecraft

12. Wang Wanjun, Gu Zhou, Xu Yiquan. Review of electrical simulation of mechanical moment of inertia in dynamic test [J]. Mechanical science and technology, 2018, 37 (12): 49-55

13. Yang Yuanqing, $\mathrm{Xu}$ Zhigang, Wang Junyi, Zhang Wei. Study on the rigid characteristics of space transfer manipulator $[\mathrm{J}]$. Journal of weapon equipment engineering, 2019, 40 (05): 100-104

14. Zhang Wei, Bai Xinlin, Xu Zhigang. Ground simulation research on the moment of inertia of space transfer manipulator [J]. Mechanical design and manufacturing, 2018

15. Cai Zixing, Xie bin. Robotics [M]. 3. Tsinghua University Press, 2015

16. Zhang Dingguo. Recursive Lagrange dynamics modeling and Simulation of multi bar space flexible robot [J]. Applied Mathematics and mechanics, 2009, 30 (10) 
17. Zhao Wuyun, Shi Zenglu, Dai Fei, Zhang Xuejun. Adams 2013 basic and application case course [M]. 1. Tsinghua University Press, 2015

18. Liu Hao, Han Jing. Matlab r2018a fully self-study yiben Tong [M]. Published by Electronic Industry Press, 2018 